\title{
COMPACTIFYING THE JACOBIAN
}

\author{
BY ALLEN B. ALTMAN AND STEVEN L. KLEIMAN ${ }^{1}$
}

Communicated by S. S. Shatz, June 28, 1976

Compactifications of jacobians of integral curves and their compatibility with specialization were considered by Igusa (Amer. J. Math. 1956) for members of a Lefschetz pencil, by Mayer-Mumford (Woods Hole, 1964) for any integral curve in terms of the moduli of torsion-free sheaves of rank 1, and by D'Souza (Bombay thesis, 1973, being improved for Astérisque) who proved the "Abel map" from the Hilbert scheme smooth where it should be for a Gorenstein curve and the compactification irreducible for a curve with nodes. Up to now, it has been impossible to make Grothendieck's fine theory (FGA) of the Picard scheme compactify the jacobian; we announce what we feel is a conceptual and technical breakthrough.

Let $f: X \rightarrow S$ be a projective morphism of locally noetherian schemes. The heart of our theory is a good notion of the functor of linear systems: for $I, F$ coherent on $X$ with $F S$-flat, set

$$
\operatorname{Lin~Sys~}_{I, F}(T)=\left\{0 \rightarrow I(G) \rightarrow F_{T} \rightarrow G \rightarrow 0 \in \operatorname{Quot}_{F / X / S}(T) \mid I(G) \simeq I_{T} \otimes N\right.
$$

for some invertible sheaf $N$ on $T$ \}.

The functor is representable ((1) below) by a manageable scheme if $I$ is $S$-simple, i.e., $S$-flat with $0_{S} \rightarrow f_{*} \operatorname{Hom}(I, I)$ universally an isomorphism. (1) is easy to prove using $\left(\right.$ EGAIII $\left._{2}, 7.7 .9\right)$ : There exists a coherent sheaf $H=H(I, F)$ on $S$ and an element $h=h(I, F)$ of $\operatorname{Hom}_{X}(I, F \otimes H)$ such that for every quasicoherent sheaf $M$ on an $S$-scheme $T$ the Yoneda map is an isomorphism

$$
y\left(h_{T}\right): \operatorname{Hom}_{T}\left(H_{T}, M\right) \stackrel{\sim}{\longrightarrow} \operatorname{Hom}_{X_{T}}\left(I_{T}, F_{T} \otimes M\right) .
$$

(1) THEOREM. If I is S-simple, then Lin Sys ${ }_{I, F}$ is universally represented by an open subscheme $U$ of $P=P(H(I, F))$, and $U$ is all of $P$ if and only if, for every geometric point $s$ of $S$, every nonzero map $I(s) \rightarrow F(s)$ is actually injective.

Assume $f$ flat with geometric fibers integral curves of arithmetic genus $p_{a}$. Our form of the existence theorems are (2), (3) below. (3) is proved via $g$ rigidification. Set

AMS (MOS) subject calssifications (1970). Primary 14D20; Secondary 14H40, 14 C10.

${ }^{1}$ Partially supported by NSF-P-42656 and by Danish NSRC No. 511-5684. Guest at Caltech, at UC Irvine, at U Copenhagen, and at U Aarhus. 
$\operatorname{Pic}_{X / S}^{-}(T)=\left\{T\right.$-flat, coherent sheaves on $X_{T}$ with torsion-free, rank-1 fibers\}/isomorphism, $\operatorname{Pic}(T)$.

(2) THEOREM. $\mathrm{Pic}_{X / S}^{-}$is separated for the étale topology, and the associated sheaf is representable by a disjoint union of projective $S$-schemes $P_{n}$. The scheme $P_{n}$ parametrizes the sheaves with Euler characteristic $n$.

(3) THEOREM. If the smooth locus of $f$ admits a section $g$, then $\mathrm{Pic}_{\bar{X} / S}$ itself is representable and there is a universal sheaf $I$.

A major feature of our theory is a very strong form (4) of Abel's theorem. It deals with F-pseudo-divisors, i.e., exact sequences $0 \rightarrow I(G) \rightarrow F \rightarrow G \rightarrow 0$ with $G$ flat and the fibers of $I(G)$ torsion-free, rank-1. These are parametrized by an open subset $P \cdot \operatorname{div}_{F / X / S}$ of Quot $F / X / S$, and it maps canonically into the compactified jacobian. Abel's theorem says this "Abel map" is the structure map of a nice family of projective spaces.

(4) THEOREM. If a universal sheaf I exists, then

$$
P-\operatorname{div}_{F / X / S}=\mathbf{P}(H(I, F)) \text {. }
$$

(4) is a direct consequence of (1). Note $I$ is simple since its geometric fibers are. This is a general principle, part of a base-change theory for Ext's. The latter can be derived from Bergman-Ogus (Proc. Amer. Math. Soc. 31 (1972), 67-74).

By (4) the Abel map is smooth over a point $p \in P_{n}$ if $H(I, F)$ is free at $p$. By base-change theory, this holds if $\operatorname{Ext}_{X(p)}^{1}(I(p), F(p))$ is 0 . If $F$ is the dualizing sheaf $\omega$, then the Ext is just $H^{0}(I(p))^{*}$, so 0 for $n<1-p_{a}$. Hence $P_{n}$ for $m=p_{a}-1-n>2 p_{a}-2$ is the quotient of Quot ${ }_{\omega / X / S}^{m}$ by a smooth and proper equivalence relation. In fact $P_{n}$ is constructed by forming this quotient. If $X$ is Gorenstein, then $\omega$ is invertible and Quot ${ }_{\omega / X / S}^{m}$ is isomorphic to $\mathrm{Hilb}_{X / S}^{m}$. In general, however, the right source of torsion-free sheaves of rank 1 is the family of submodules of $\omega$. This observation came naturally but is a real surprise.

As to the irreducibility of $P_{n}$, Iarrobino helped prove (5) below and find an example showing how sharp (5) is. (5) fails for a complete intersection in $\mathbf{P}^{3}$ over a field.

(5) THEOREM. If $X$ lies on a smooth projective $S$-scheme of relative dimension 2, then each $P_{n}$ is $S$-flat and its geometric fibers are integral locally complete intersections of dimension $p_{a}$.

The proofs of (2), (3), (4) require not that $X / S$ have relative dimension 1 , but that its fibers $X(s)$ be Cohen-Macaulay (so a dualizing sheaf $\omega$ exists) and that the torsion-free sheaves $J$ on them be Cohen-Macaulay (so $\operatorname{Ext}_{X(s)}^{1}(J, \omega(s)(m)$ ) is 0 for $m \gg 0$ ). However, the enlarged Picard scheme constructed this way is not $S$-compact. 
A variant of the theory represents $\operatorname{Pic}_{X / S \text {;ét }}^{-}$by a disjoint union of proper algebraic $S$-spaces for $f$ flat with integral geometric fibers. In fact, with no special hypotheses on $f$, it yields a strong, new result (6), the representability of the moduli functor of simple sheaves:

$$
\operatorname{Spl}_{X / S}(T)=\left\{T \text {-simple sheaves on } X_{T}\right\} / \text { isomorphism, } \operatorname{Pic}(T) \text {. }
$$

(6) THEOREM. $\mathrm{Spl}_{X / S}$ is separated for the étale topology, and the associated étale sheaf is representable by an (unseparated) algebraic space locally of finite type over $S$.

The proof involves no tedious checking of axioms, just forming a quotient by a smooth equivalence relation (Artin, Invent. Math. 27 (1974), p. 184. Lemma (6.4)). The simple sheaves are viewed as quotients of certain sheaves, and the equivalence relation is analyzed remarkably like the way linear equivalence was.

DEPARTMENTO DE MATEMATICO, UNIVERSIDAD DE SIMÓN BOLÍVAR, APARTADO POSTAL 5354 CARACAS, VENEZUELA

DEPARTMENT OF MATHEMATICS, MASSACHUSETTS INSTITUTE OF TECHNOLOGY, CAMBRIDGE, MASSACHUSETTS 02139 